\title{
Prognostic insights of molecular imaging in cardiac sarcoidosis
}

\author{
Riccardo Laudicella, MD (1), ${ }^{\mathrm{a}}$ Fabio Minutoli, $M D{ }^{\mathrm{a}}$ and Sergio Baldari, $\mathrm{MD}^{\mathrm{a}}$ \\ a Department of Biomedical and Dental Sciences and of Morpho-functional Imaging, Nuclear \\ Medicine Unit, University of Messina, Messina, ME, Italy
}

Received Mar 19, 2019; accepted Mar 19, 2019

doi: 10.1007/s12350-019-01701-3

\section{See related article, pp. 199-205}

Sarcoidosis is a chronic granulomatous disorder characterized by a multisystem evolution with an unknown etiology, even if it is believed to be due to an exaggerated cellular immune response by the helper/ inducer $\mathrm{T}$ lymphocytes to exogenous or auto-antigens. This disorder can potentially affect any organ: the most common sites are lymph nodes (especially intra-thoracic), lungs, liver, spleen, skin, and eyes. Sarcoidosis occurs most commonly in Scandinavian areas and in young adults, whilst clinically the more severe forms are reported in the black population. In $25-30 \%$, sarcoidosis may involve the myocardium and its elective localization is represented by the interventricular septum. ${ }^{1}$ Usually, the clinical consequences of cardiac sarcoidosis (CS) are conduction/rhythm disturbances and, in case of massive myocardial involvement, heart failure and death. ${ }^{2}$ Cardiac involvement of sarcoidosis has a pivotal role in the assessment of prognosis and contributes to a major part of the disease morbidity and mortality. ${ }^{3}$ The gold standard for the diagnosis of CS is considered the endomyocardial biopsy (EMB): however, this invasive technique is characterized by potential complications ${ }^{4}$ and low sensitivity ( $<60 \%$, even if repeated) in determining the non-caseating sarcoid granuloma, due to possible sampling errors. ${ }^{5}$ Therefore, the diagnosis of CS can be made on the basis of imaging and clinical features in addition to extracardiac biopsy evidence of

Reprint requests: Riccardo Laudicella, MD, Department of Biomedical and Dental Sciences and of Morphofunctional Imaging, Nuclear Medicine Unit, University of Messina, Via Consolare Valeria n.1, Messina, ME, Italy; riclaudi@hotmail.it

J Nucl Cardiol 2021;28:206-8.

$1071-3581 / \$ 34.00$

Copyright (C) 2019 American Society of Nuclear Cardiology. sarcoidosis. Consequently, radiological evaluation in suspected CS is nowadays largely required. ${ }^{6}$ Different features can be evaluated, and among them, the most relevant are myocardial fibrosis and inflammation. Indeed, the myocardial late-gadolinium enhancement (LGE) due to scar or edema in cardiac magnetic resonance imaging (CMRI) is considered a diagnostic criterion for CS., ${ }^{6,7}$ Fluorine-18 labeled 2-deoxy-2-fluoro-D-glucose positron emission computer tomography $\left({ }^{18} \mathrm{~F}-\mathrm{FDG}-\mathrm{PET} / \mathrm{CT}\right)$ shows high accuracy ${ }^{8}$ and is widely used in the evaluation of CS activity (especially for active inflammation assessment), even in the prediction of adverse events. ${ }^{9}$ Obtaining a complete FDG-uptake suppression in normal myocardium through dietary protocols permits a more confident diagnosis of cardiac involvement; so it is important to check the rate of cardiac suppression obtained in own Center and modify/ lengthen the dietary protocol, accordingly. Indeed, although several patterns of normal left-ventricular (LV) FDG-uptake have been described, ${ }^{10,11}$ non-homogeneous cardiac distribution of radiotracer may rise interpretative doubts; thus, a complete and robust clinical assessment is mandatory to determine the most probable diagnosis. In the current issue of the Journal, Tuominen et al. performed a retrospective analysis of 137 patients who underwent ${ }^{18} \mathrm{~F}$-FDG-PET/CT imaging for suspected CS evaluation. In this cohort, 35/137 patients were already known to be affected by sarcoidosis, whilst 18/137 (13\%) have been diagnosed with CS (confirmed by EMB in 7). They focused the attention in the prediction of cardiovascular events (defined as a reduction in $\mathrm{LV}$ ejection fraction, hospitalization due to cardiac arrhythmia and death) of either quantitative FDG-PET parameters as of the cardiac pathological pattern of FDG-uptake. In this paper, a relevant prognostic value of abnormal right-ventricular (RV) FDGuptake (focal or focal on diffuse pattern) has been demonstrated in patients showing atrio-ventricular block, ventricular or supraventricular arrhythmia, 
unexplained dilated cardiomyopathy, unexplained low ejection fraction (EF) on echocardiography, echocardiographic signs of cardiac sarcoidosis, or syncope. In the small group of patients who underwent both PET and CMRI, the positive predictive value of abnormal RV FDG-uptake was higher than the evidence of LGE at CMRI, whereas negative predictive values were similar. ${ }^{11}$ The presence of RV FDG-uptake may reflect the spread of CS activity, being a predictor of death ${ }^{12}$ or ventricular tachycardia events. ${ }^{13}$ In the cohort of Tuominen, cardiac events were not significantly more common in patients with diagnosed CS than those without: unlike RV FDG-uptake, cardiac PET positivity by itself was not predictive of cardiac events, and cardiologists should be aware of this clinical implication. It should be remembered that heart failure can be due to an increase in pulmonary vascular resistance secondary to diffuse interstitial fibrosis: in these cases, the picture of the chronic pulmonary heart is realized. In this sense, cardiac involvement should be detected as soon as possible in order to establish an early treatment. However, the main findings reported by Tuominen and Colleagues was that, in survival analysis, a total cardiac metabolic activity (tCMA) value of more than $900 \mathrm{MBq}$ significantly predicted cardiac events $(\mathrm{p}=0.032)$ and that pathological RV FDG-uptake was more common in patients with cardiovascular events at follow-up than those without $(\mathrm{p}=0.001)$. Indeed, the association between a high tCMA and RV pathological FDG-uptake should be considered as a significant risk marker in patients with suspected CS. ${ }^{11}$ In accordance with these results, Ahmandian et al. ${ }^{14}$ showed that a high tCMA was an independent predictor of cardiovascular events in CS patients. Similarly, in a recent study by Omote et al., CS patients with RV FDG-uptake showed notably higher cardiac metabolic volume (CMV), total lesion glycolysis (TLG), LV standardized-uptake-value (SUV) max, LV involvement, and cardiac adverse events than those without. RV FDG-uptake would indicate the spread of disease activity into the whole heart, the advanced stages of sarcoidosis, possibly predicting positive findings of EMB. ${ }^{15}$ Blankstein et al. ${ }^{13}$ showed that RV FDG-uptake in suspected CS patients, was related to a five-time improved risk for cardiovascular events, whilst Flores et al. ${ }^{16}$ demonstrated a strong correlation between a high SUV at the time of diagnosis of CS (especially in basal segments) and future cardiac events. The quantitative variations of FDG-uptake have shown to be a valuable tool in the assessment of CS pathological evolution: in long-term follow-up CS patients, it has been demonstrated that reduction in FDG-uptake strongly correlated with reduction of major cardiac adverse events. ${ }^{17}$ Furthermore, in a study by Osborne et al. ${ }^{18}$ FDG-uptake variations in terms of metabolic activity/volume showed an inverse correlation with changes in systolic function. The information obtained by FGD-PET/CT and CMRI are usually complementary. The introduction and the desirable widespread of hybrid systems combining PET and MRI (PET/MRI) may be a real opportunity for CS imaging thus improving the imaging performance in this topic. ${ }^{9}$ Further studies evaluating the capability of ${ }^{18} \mathrm{~F}$-FDGPET in diagnosis and prognosis of cardiac disease as well as evaluation of new imaging approach-using radiopharmaceuticals as ${ }^{68}$ Gallium-DOTANOC, ${ }^{18} \mathrm{~F}$ Flurpiridaz, and ${ }^{13}$ Nitrogen-Ammonia that are currently undergoing testing and have shown promising results in initial small studies ${ }^{20}$-are warranted to establish a unified approach, thus improving the assessment of CS.

\section{Disclosure}

Laudicella R., Minutoli F. and Baldari S. have no relevant or material financial interests that can relate to the research described in this paper.

\section{References}

1. Birnie DH, Nery PB, Ha AC, Beanlands RSB. Cardiac sarcoidosis. J Am Coll Cardiol. 2016;68:411-21.

2. Valeyre D, Prasse A, Nunes H, Uzunhan Y, Brillet PY, MüllerQuernheim J. Sarcoidosis. Lancet. 2014;383:1155-67.

3. Murtagh G, Laffin LJ, Beshai JF, Maffessanti F, Bonham CA, Patel AV, et al. Prognosis of myocardial damage in sarcoidosis patients with preserved left ventricular ejection fraction: Risk stratification using cardiovascular magnetic resonance. Circ Cardiovasc Imaging. 2016;9(1):e003738.

4. Yilmaz A, Kindermann I, Kindermann M, Mahfoud F, Ukena C, Athanasiadis A, et al. Comparative evaluation of left and right ventricular endomyocardial biopsy: Differences in complication rate and diagnostic performance. Circulation. 2010;122:900-9.

5. Simonen P, Lehtonen J, Kupari M. Long-term outcome in probable versus absolute cardiac sarcoidosis. Am J Cardiol. 2019;123:674-8.

6. Terasaki F, Yoshinaga K. New guidelines for diagnosis of cardiac sarcoidosis in Japan. Ann Nucl Cardiol. 2017;3:42-5.

7. Ordovas KG, Higgins CB. Delayed contrast enhancement on MR images of myocardium: Past, present, future. Radiology. 2011;261:358-74.

8. Youssef G, Leung E, Mylonas I, Nery P, Williams K, Wisenberg $\mathrm{G}$, et al. The use of 18F-FDG PET in the diagnosis of cardiac sarcoidosis: A systematic review and metaanalysis including the Ontario experience. J Nucl Med. 2012;53:241-8.

9. Mc Ardle BA, Birnie DH, Klein R, de Kemp RA, Leung E, Renaud J, et al. Is there an association between clinical presentation and the location and extent of myocardial involvement of cardiac sarcoidosis as assessed by ${ }^{18} \mathrm{~F}$-fluorodoexyglucose positron emission tomography? Circ Cardiovasc Imaging. 2013;6:617-26.

10. Betancourt Cuellar SL, Palacio D, Benveniste MF, Carter BW, Gladish G. Pitfalls and misinterpretations of cardiac findings on PET/CT Imaging: A careful look at the heart in oncology patients. Curr Probl Diagn Radiol. 2019;48:172-83. 
11. Tuominen H, Haarala A, Tikkakoski A, Kähönen M, Nikus K, Sipilä K. FDG-PET in possible cardiac sarcoidosis: Right ventricular uptake and high total cardiac metabolic activity predict cardiovascular events. J Nucl Cardiol. 2019. https://doi.org/10.10 07/s12350-019-01659-2.

12. Tavora F, Cresswell N, Li L, Ripple M, Solomon C, Burke A. Comparison of necropsy findings in patients with sarcoidosis dying suddenly from cardiac sarcoidosis versus dying suddenly from other causes. Am J Cardiol. 2009;104:571-7.

13. Blankstein R, Osborne M, Naya M, Waller A, Kim CK, Murthy $\mathrm{VL}$, et al. Cardiac positron emission tomography enhances prognostic assessments of patients with suspected cardiac sarcoidosis. J Am Coll Cardiol. 2014;63:329-36.

14. Ahmadian A, Brogan A, Berman J, Sverdlov AL, Mercier G, Mazzini M, et al. Quantitative interpretation of FDG PET/CT with myocardial perfusion imaging increases diagnostic information in the evaluation of cardiac sarcoidosis. J Nucl Cardiol. 2014;21:92539.

15. Omote K, Naya M, Koyanagawa K, Aikawa T, Manabe O, Nagai $\mathrm{T}$, et al. (18)F-FDG uptake of the right ventricle is an important predictor of histopathologic diagnosis by endomyocardial biopsy in patients with cardiac sarcoidosis. J Nucl Cardiol. 2019. https://d oi.org/10.1007/s12350-018-01541-7.

16. Flores RJ, Flaherty KR, Jin Z, Bokhari S. The prognostic value of quantitating and localizing F-18 FDG uptake in cardiac sarcoidosis. J Nucl Cardiol. 2018. https://doi.org/10.1007/s12350018-01504-y.

17. Muser D, Santangeli P, Castro SA, Liang JJ, Enriquez A, Werner TJ, et al. Prognostic role of serial quantitative evaluation of (18)Ffluorodeoxyglucose uptake by PET/CT in patients with cardiac sarcoidosis presenting with ventricular tachycardia. Eur $\mathrm{J}$ Nucl Med Mol Imaging. 2018;45:1394-404

18. Osborne MT, Hulten EA, Singh A, Waller AH, Bittencourt MS, Stewart GC, et al. Reduction in ${ }^{18} \mathrm{~F}$-fluorodeoxyglucose uptake on serial cardiac positron emission tomography is associated with improved left ventricular ejection fraction in patients with cardiac sarcoidosis. J Nucl Cardiol. 2014;21:166-74.

19. Wicks EC, Menezes LJ, Barnes A, Mohiddin SA, Sekhri N, Porter JC, et al. Diagnostic accuracy and prognostic value of simultaneous hybrid $18 \mathrm{~F}$-fluorodeoxyglucose positron emission tomography/magnetic resonance imaging in cardiac sarcoidosis. Eur Heart J Cardiovasc Imaging. 2018;19:757-67.

20. Yatsynovich Y, Valencia D, Petrov M, Linares JD, Rahman MM, Dittoe N. Updates on the role of imaging in cardiac sarcoidosis. Curr Treat Options Cardiovasc Med. 2018;20:74.

Publisher's Note Springer Nature remains neutral with regard to jurisdictional claims in published maps and institutional affiliations. 